\section{OP0115 EFFECT OF NINTEDANIB ON PROGRESSION OF INTERSTITIAL LUNG DISEASE (ILD) IN PATIENTS WITH AUTOIMMUNE DISEASE-RELATED ILDS: FURTHER DATA FROM THE INBUILD TRIAL}

E. Matteson ${ }^{1}$, C. Kelly ${ }^{2}$, J. Distler ${ }^{3}$, A. M. Hoffmann-Vold ${ }^{4}$, J. Seibold ${ }^{5}$, S. Mittoo ${ }^{6}$, O. Distler ${ }^{7}$, P. F. Dellaripa ${ }^{8}$, A. James ${ }^{9}$, R. Schlenker-Herceg ${ }^{10}$, S. Stowasser ${ }^{9}$, M. Quaresma ${ }^{9}$, K. R. Flaherty ${ }^{11} .{ }^{1}$ Mayo Clinic College of Medicine and Science, Rochester, Minnesota, United States of America; ${ }^{2}$ Institute of Cellular Medicine, Newcastle University, Newcastle upon Tyne, United Kingdom; ${ }^{3}$ University of Erlangen-Nuremberg, Erlangen, Germany; ${ }^{4}$ Department of Rheumatology, Oslo University Hospital, Oslo, Norway; ${ }^{5}$ Scleroderma Research Consultants LLC, Aiken, South Carolina, United States of America; ${ }^{6}$ University Health Network, Toronto, Ontario, Canada; ${ }^{7}$ Department of Rheumatology, University Hospital Zurich, Zurich, Switzerland; ${ }^{8}$ Division of Rheumatology, Brigham and Women's Hospital, Boston, MA, United States of America; ${ }^{9}$ Boehringer Ingelheim International $\mathrm{GmbH}$, Ingelheim am Rhein, Germany; ${ }^{10}$ Boehringer Ingelheim Pharmaceuticals, Inc., Ridgefield, CT, United States of America; ${ }^{11}$ Division of Pulmonary and Critical Care Medicine, University of Michigan, Ann Arbor, MI, United States of America

Background: In the INBUILD trial in patients with progressive fibrosing ILDs, nintedanib reduced the rate of decline in forced vital capacity (FVC) versus placebo over 52 weeks both in the overall population and in the subgroup with autoimmune disease-related ILDs. Patients continued blinded randomised treatment until the end of the trial.

Objectives: Assess the effects of nintedanib on the risks of death, acute exacerbation of ILD or death, and disease progression or death over the whole INBUILD trial in patients with autoimmune disease-related ILDs and a progressive phenotype.

Methods: Patients with progressive fibrosing ILDs other than idiopathic pulmonary fibrosis (IPF) were randomised to receive nintedanib $150 \mathrm{mg}$ bid or placebo. Time to i) death, ii) first acute exacerbation of ILD or death, and iii) disease progression (absolute decline in FVC $\geq 10 \%$ predicted) or death, over the whole trial were analysed in patients with autoimmune disease-related ILDs. Incidence rates of adverse events per 100 patient-years were calculated based on events with onset between the first trial drug intake and the last intake plus 28 days. Analyses were descriptive.

Results: Of 663 patients, 170 (82 nintedanib, 88 placebo) had autoimmune disease-related ILDs (89 RA-ILD, 39 SSc-ILD, 19 MCTD-ILD, 23 other autoimmune ILDs including Sjogren's disease-related ILD [ $n=7]$, interstitial pneumonia with autoimmune features [ $n=5]$ and undifferentiated CTD-ILD [ $n=3]$ ). Over the whole trial, in the nintedanib and placebo groups, respectively, mean (SD) exposure to drug was 15.4 (7.4) and 16.9 (6.1) months and maximum exposure was 26.0 and 25.2 months; $62(75.6 \%)$ and 68 (77.3\%) patients in these groups, respectively, completed the planned observation time. Over the whole trial, in the nintedanib and placebo groups, respectively, $9.8 \%$ and $12.5 \%$ of patients died, $12.2 \%$ and $20.5 \%$ of patients had $\geq 1$ acute exacerbation of ILD or died, and $40.2 \%$ and $53.4 \%$ of patients had disease progression or died (Table). Diarrhoea was the most common adverse event, with incidence rates of 139.2 and 26.3 events per 100 patient-years in the nintedanib and placebo groups, respectively. Adverse events led to treatment discontinuation in $20.7 \%$ of patients in the nintedanib group and $13.6 \%$ of patients in the placebo group.

Conclusion: Data from the INBUILD trial suggest that nintedanib has a clinically meaningful effect on slowing the progression of ILD in patients with progressive fibrosing autoimmune disease-related ILDs, with adverse events that can be tolerated by most patients.

Table.

\begin{tabular}{lccc}
\hline & $\begin{array}{c}\text { Nintedanib } \\
(\mathbf{n}=\mathbf{8 2})\end{array}$ & $\begin{array}{c}\text { Placebo } \\
(\mathbf{n}=\mathbf{8 8})\end{array}$ & HR (95\% Cl)* \\
\hline Death & $8(9.8)$ & $11(12.5)$ & $0.80(0.32,1.98)$ \\
$\geq 1$ acute exacerbation of ILD or death & $10(12.2)$ & $18(20.5)$ & $0.58(0.27,1.27)$ \\
Disease progression (absolute decline in & $33(40.2)$ & $47(53.4)$ & $0.72(0.46,1.13)$ \\
FVC $\geq 10 \%$ predicted) or death & & &
\end{tabular}

$\mathrm{n}(\%)$ with event over the whole trial (mean [SD] exposure: 15.4 [7.4] and 16.9 [6.1] months in nintedanib and placebo groups, respectively). *Based on time to first event.

Disclosure of Interests: Eric Matteson Grant/research support from: Pfizer, Consultant of: Boehringer Ingelheim, Gilead, TympoBio, Arena Pharmaceuticals, Speakers bureau: Simply Speaking, Clive Kelly Consultant of: Boehringer Ingelheim, Speakers bureau: Boehringer Ingelheim, Jörg Distler Grant/research support from: Boehringer Ingelheim, Consultant of: Boehringer Ingelheim, Paid instructor for: Boehringer Ingelheim, Speakers bureau: Boehringer Ingelheim, Anna-Maria Hoffmann-Vold Grant/research support from: Boehringer Ingelheim,
Consultant of: Boehringer Ingelheim, Actelion, Bayer, GlaxoSmithKline, Speakers bureau: Boehringer Ingelheim, Actelion, Roche, James Seibold Shareholder of: BriaCell, Pacific Therapeutics, Consultant of: Atlantic, Blade Therapeutics, Eicos Sciences, Eiger Biopharmaceuticals, Indalo Therapeutics, Mitsubishi Tanabe Pharma, Bayer, Xenikos, Boehringer Ingelheim, Camurus, Corbus Pharmaceuticals, EMD Serono, Speakers bureau: Boehringer Ingelheim, Shikha Mittoo Grant/research support from: Pfizer, Consultant of: Novartis, Abbvie, Pfizer, Oliver Distler Grant/research support from: Grants/Research support from Actelion, Bayer, Boehringer Ingelheim, Competitive Drug Development International Ltd. and Mitsubishi Tanabe; he also holds the issued Patent on mir-29 for the treatment of systemic sclerosis (US8247389, EP2331143)., Consultant of: Consultancy fees from Actelion, Acceleron Pharma, AnaMar, Bayer, Baecon Discovery, Blade Therapeutics, Boehringer, CSL Behring, Catenion, ChemomAb, Curzion Pharmaceuticals, Ergonex, Galapagos NV, GSK, Glenmark Pharmaceuticals, Inventiva, Italfarmaco, iQvia, medac, Medscape, Mitsubishi Tanabe Pharma, MSD, Roche, Sanofi and UCB, Speakers bureau: Speaker fees from Actelion, Bayer, Boehringer Ingelheim, Medscape, Pfizer and Roche, Paul F. Dellaripa Grant/research support from: Paul Dellaripa has received institutional grants from Genentech, Consultant of: Paul Dellaripa participated in advisory boards for Boehringer Ingelheim, Alexandra James Employee of: Employee of Boehringer Ingelheim, Rozsa Schlenker-Herceg Employee of: Employee of Boehringer Ingelheim, Susanne Stowasser Employee of: Employee of Boehringer Ingelheim, Manuel Quaresma Employee of: Employee of Boehringer Ingelheim, Kevin R. Flaherty Grant/research support from: Kevin Flaherty has received grants from Boehringer Ingelheim, Consultant of: Kevin Flaherty has acted as a consultant for Boehringer Ingelheim, Bellerophon, Blade Therapeutics, Roche/Genentech, and Veracyte

He was a member of the INBUILD trial Steering Committee

DOI: 10.1136/annrheumdis-2020-eular.3211

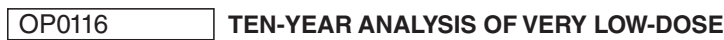 GLUCOCORTICOIDS IN EARLY RA (ESPOIR COHORT) SUPPORTS A TIME-DEPENDENT RISK OF SEVERE} OUTCOMES

C. Roubille ${ }^{1,2}$, A. Coffy ${ }^{3}$, N. Rincheval ${ }^{3}$, M. Dougados ${ }^{4}$, R. M. Flipo ${ }^{5}$, J. P. Daures ${ }^{3}$, B. Combe ${ }^{6} .{ }^{1}$ Montpellier University Hospital, Department of Internal Medicine, Montpellier, France; ${ }^{2}$ Laboratoire PHYMEDEXP, INSERM U1046, CNRS UMR 9214, University of Montpellier, Montpellier, France; ${ }^{3}$ Statistiques, University Institute of Clinical Research, EA2415, Montpellier, France; ${ }^{4}$ Paris Descartes University, Rheumatology Department, Cochin Hospital, AP-HP, Paris, Paris, France; ${ }^{5}$ Roger Salengro Hospital, Rheumatology Department, Lille, France; ${ }^{6}$ Montpellier University Hospital, Lapeyronie Hospital, Rheumatology Department, Montpellier, France

Background: We previously failed to find any significant difference with regard to severe outcomes (death, severe infections, fractures, cardiovascular diseases [CVD]) between recent-onset RA patients taking or not low-dose GC treatment in a 7-year analysis of the ESPOIR cohort (1).

Objectives: To explore the 10-year tolerability profile of GC use in patients with early RA.

Methods: We analysed data from the early arthritis (less than 6 months disease duration) ESPOIR cohort. Patients were stratified in two groups, with or without GC treatment at least once during their follow-up (median 10 years IQR [9-10]). The primary outcome was a composite of death, CVD (including myocardial ischemia, cerebrovascular accident and heart failure), severe infection and fracture. In order to reduce the impact of treatment selection bias and potential confounding factors, the weighted Cox time-dependent analysis model was used with inverse probability of treatment weighting (IPTW) propensity score method. Results: Among the 608 RA patients (480 women, mean age of $47.5 \pm 12.1$ years), 397 patients $(65 \%)$ received low-dose prednisone (median $1.9 \mathrm{mg} /$ day [IQR 0.6-4.2], mainly during the first 6 months $(70 \%)$. The mean duration of GC treatment was 44.6 months \pm 40.1 . Overall, 95 events were identified during follow-up: 10 deaths, $18 \mathrm{CVD}, 32$ fractures and 35 severe infections. Based on univariate analysis at 10 years, patients taking GC experienced significantly more events $(n=71)$ than those without $G C(n=24)(p=0.035)$, especially severe infections ( $n=30$ with $\mathrm{GC}$ versus 5 without $\mathrm{GC}, \mathrm{p}=0.009$ ) (table 1 ), with a cumulative dose effect $(\mathrm{p}=0.007)$. On weighted Cox time-dependent analysis, using the IPTW propensity score method, the risk of events over time was significantly associated with GC treatment ( $p<0.001)$, age, history of hypertension and erythrocyte sedimentation rate. The risk associated with GC treatment, estimated by the hazard ratio (HR), increased between the first follow-up visit (HR at 6 months $=0.39,95 \% \mathrm{Cl} 0.19-0.82)$ and 10 years $(H R=6.83,95 \% \mathrm{Cl} 2.29-20.35)$ (figure 1 and table 2). 
Table 1. Primary outcome and events at 10 years: univariate analysis

\begin{tabular}{lcccc}
\hline & Total study population ( $\mathrm{n}=608)$ & Without GC & With CG & P Value \\
\hline Primary outcome & $95(15.6 \%)$ & $24(11.4 \%)$ & $71(17.9 \%)$ & 0.035 \\
Death & $10(1.6 \%)$ & $1(0.5 \%)$ & $9(2.3 \%)$ & 0.103 \\
Cardiovascular diseases & $18(3 \%)$ & $3(1.4 \%)$ & $15(3.8 \%)$ & 0.177 \\
Severe infections & $35(5.8 \%)$ & $5(2.4 \%)$ & $30(7.6 \%)$ & 0.009 \\
Fractures & $32(5.3 \%)$ & $15(7.1 \%)$ & $17(4.3 \%)$ & 0.137 \\
\hline
\end{tabular}

Table 2. Time-dependent relationship between glucocorticoids treatment and risk of events estimated by hazard ratio

\begin{tabular}{lc}
\hline Time (Months) & Hazard Ratio $(95 \% \mathrm{Cl})$ \\
\hline 12 & $0.46(0.23-0.90)$ \\
24 & $0.62(0.36-1.08)$ \\
36 & $0.83(0.52-1.33)$ \\
48 & $1.12(0.73-1.72)$ \\
60 & $1.52(0.96-2.40)$ \\
72 & $2.05(1.19-3.52)$ \\
84 & $2.77(1.44-5.34)$ \\
96 & $3.74(1.69-8.26)$ \\
108 & $5.05(1.98-12.91)$ \\
\hline
\end{tabular}

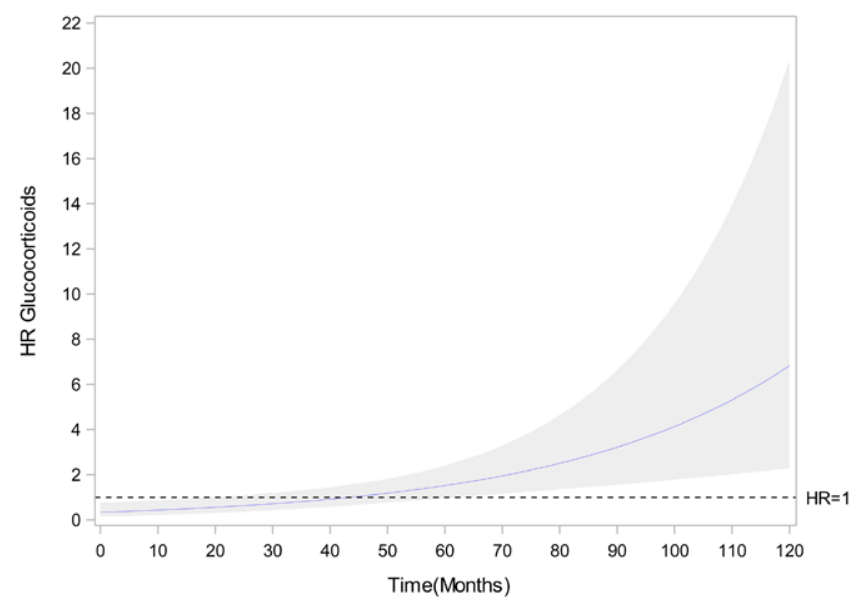

Figure 1. Time-dependent relationship between glucocorticoids treatment and risk of events estimated by hazard ratio (HR)

Conclusion: This 10-year analysis of the ESPOIR cohort supports a dose and time-dependent impact of very low-dose GC treatment in early RA, with a longterm high risk of severe outcomes.

Disclosure of Interests: Camille Roubille Consultant of: Servier, Pfizer, Novartis, Amandine Coffy: None declared, Nathalie Rincheval: None declared, Maxime Dougados Grant/research support from: AbbVie, Eli Lilly, Merck, Novartis, Pfizer and UCB Pharma, Consultant of: AbbVie, Eli Lilly, Merck, Novartis, Pfizer and UCB Pharma, Speakers bureau: AbbVie, Eli Lilly, Merck, Novartis, Pfizer and UCB Pharma, Rene-Marc Flipo Speakers bureau: Novartis, Janssen, Lilly, JeanPierre Daures: None declared, Bernard Combe Grant/research support from: Novartis, Pfizer, Roche-Chugai, Consultant of: AbbVie; Gilead Sciences, Inc.; Janssen; Eli Lilly and Company; Pfizer; Roche-Chugai; Sanofi, Speakers bureau: Bristol-Myers Squibb; Gilead Sciences, Inc.; Eli Lilly and Company; Merck Sharp \& Dohme; Pfizer; Roche-Chugai; UCB

DOI: 10.1136/annrheumdis-2020-eular.3917

\section{OP0117 LONGITUDINAL CHANGE IN THE CENTRAL NERVOUS SYSTEM PAIN RESPONSE AFTER TREATMENT WITH CERTOLIZUMAB OR PLACEBO. A POST-HOC ANALYSIS FROM THE PRECEPRA TRIAL}

J. Rech ${ }^{1}$, K. Tascilar ${ }^{1}$, H. Schenker ${ }^{1}$, M. Hagen ${ }^{1}$, M. Sergeeva ${ }^{2}$ M. Selvakumar ${ }^{2}$, L. Konerth ${ }^{2}$, J. Prade $^{2}$, S. Strobelt ${ }^{2}$, V. Schönau ${ }^{1}$, L. Valor ${ }^{1}$, A. Hueber ${ }^{3}$, D. Simon ${ }^{1}$, A. Kleyer ${ }^{1}$, F. Behrens ${ }^{4}$, C. Baerwald ${ }^{5}$, S. Finzel ${ }^{6}$, R. Voll ${ }^{6}$, E. Feist ${ }^{7}$, J. A. P. Da Silva ${ }^{8}$, A. Doerfler ${ }^{9}$, N. Damjanov ${ }^{10}$, A. Hess ${ }^{2}$, G. Schett ${ }^{1} .{ }^{1}$ Friedrich-Alexander University (FAU) Erlangen-Nürnberg and Universitätsklinikum Erlangen, Department of Internal Medicine 3 Rheumatology and Immunology, Erlangen, Germany; ${ }^{2}$ Friedrich-Alexander University (FAU) Erlangen-Nürnberg, Institute for Experimental Pharmacology,
Erlangen, Germany; ${ }^{3}$ Sozialstiftung Bamberg, Section Rheumatology, Bamberg, Germany; ${ }^{4}$ Goethe University Frankfurt, Rheumatology and Fraunhofer TMP Frankfurt, Germany; ${ }^{5}$ Universitätsklinikum Leipzig, Medizinische Klinik III Bereich Rheumatologie, Leipzig, Germany; ${ }^{6}$ Universitätsklinikum Freiburg, Klinik für Rheumatologie und Klinische Immunologie, Freiburg, Germany; ${ }^{7}$ Klinik für Rheumatologie der Helios Fachklinik, Vogelsang-Gommern, Germany; ${ }^{8}$ Hospitais da Universidade (SRHUC), Department Reumatologia, Coimbra, Portugal; ${ }^{9}$ Friedrich-Alexander-University Erlangen-Nürnberg ( $F A U)$, Universitätsklinikum Erlangen, Abteilung für Neuroradiologie, Erlangen, Germany; ${ }^{10}$ Belgrade University School of Medicine, Institute of Rheumatology, Belgrade, Serbia

Background: Tumor necrosis factor inhibitors have revolutionized the treatment of rheumatoid arthritis (RA). However, only about $50 \%$ of the patients respond well to TNF inhibitors. Therefore, markers that predict response to TNF inhibitors are valuable. Previously we demonstrated that central nervous system (CNS) response to nociceptive stimuli, measured by $\mathrm{fMRI}$ of the brain as blood oxygen level dependent (BOLD) signals, decreases already after 24 hours of anti-TNF administration a higher pre-treatment BOLD signal volume seems to predict clinical response to treatment with certolizumabpegol (CZP) ${ }^{1,2}$. We therefore hypothesized that the baseline volume of BOLD signal in the CNS could predict anti-TNF treatment response.

Objectives: To perform a randomized placebo controlled trial in active RA patients to test the effect of TNF inhibition on arthritis induced pain activ ity in the brain and to test whether patients with high-level RA-related brain activation react differently to TNF-inhibitors than patients with low-level brain activation.

Methods: Adult RA patients fulfilling the 2010 ACR/EULAR classification criteria with a DAS28 $>3.2$ receiving stable DMARD treatment for at least 3 months were eligible. Patients underwent the first $\mathrm{PMRI}$ at screening measuring BOLD signal upon MCP joint compression and were stratified into low ( $<700$ units) and high (>700 units) voxel counts. Then patients were randomized to CZP or placebo with a 2:1 ratio. The second and third fMRI were performed after 12 and 24 weeks, respectively. Control stimulation was done by measuring brain activation after non-painful finger tapping.

Results: 156 RA patients with moderate-to-high disease activity participated in the study. In the finger tapping control, fMRI showed no significant changes in BOLD signal in the CZP-L and CZP-H arms, but a slight but significant decrease $(p=0.043)$ was observed. After joint compression, the CZP-L group showed significant increase in the BOLD signal volume $(p=0.043)$ in $\mathrm{fMRI}-2$ as compared to fMRI-1 with no further significant changes. In contrast, in the CZP-H group, the BOLD signal volume significantly decreased $(p=0.037)$ in $\mathrm{fMRI}-2$ and continued
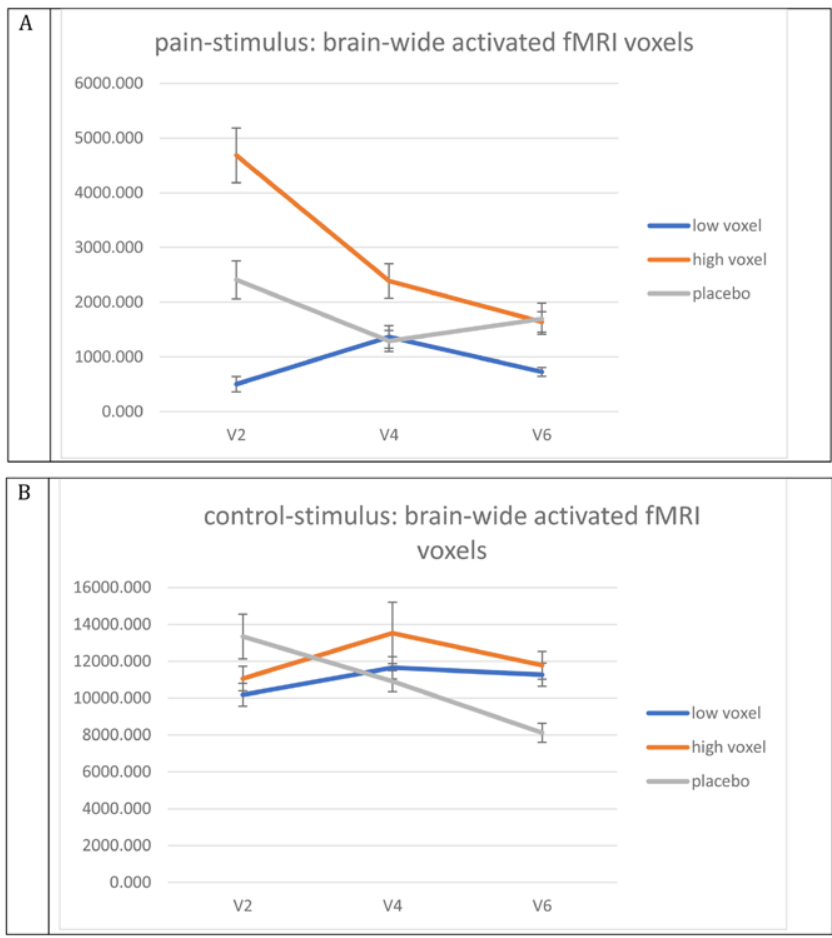

Fig 1. BOLD fMRI responses to painful stimulation 